\title{
GASEOUS ACCRETION FLOWS IN THE INNER PARSEC OF THE GALAXY
}

\author{
ROBERT COKER \\ Physics Department, The University of Arizona, \\ Tucson, AZ, 85721 USA \\ AND \\ FULVIO MELIA \\ Physics Department and Steward Observatory, \\ The University of Arizona, Tucson, AZ, 85721 USA
}

\begin{abstract}
Many characteristics of galactic nuclei may be associated with the accretion of ambient gas by a central concentration of mass. Using a 3D hydrodynamical code, we have been simulating this accretion process for Sgr A*, the compact nonthermal source at the center of the Milky Way, in order to realistically model the gaseous flows in the inner parsec of our Galaxy. In the most recent simulations, we have taken into account the multi-point-like distribution of wind sources and we find that the structure of the flow can be significantly different from that due to a uniform medium. We here present our results concerning the mass and angular momentum accretion rates and discuss how these may be used to set constraints on our Galaxy's central engine.
\end{abstract}

\section{Background}

Using the NCSA 3D hydrodynamical code ZEUS, we have undertaken numerical simulations of the gaseous flows in the central parsec of the Milky Way to determine constraints on the power source of Sgr $\mathrm{A}^{*}$, which is thought to be a $2-3 \times 10^{6} M_{\odot}$ black hole. Determining the characteristics of the gas that may be accreting onto Sgr A*, and, perhaps, forming an accretion disk, is critical to modeling its spectrum. In particular, we wish to determine what role the Galaxy's central cluster of massive wind-losing 
stars, IRS 16, plays in producing the gaseous distributions of the central cavity. Finally, we look for a way to link Sgr A* to the more active nuclei of other galaxies.

A variety of authors have confirmed that IRS 16 consists of a number of massive stars with large stellar winds, a natural power source for Sgr A*. ¿From emission line observations, the Galactic Center wind has a mass flow away from the IRS 16 region of $\sim 0.003 M_{\odot} \mathrm{yr}^{-1}$ (Geballe et. al., 1991). In the simulations presented here, in order to facilitate comparison with earlier work, we use a Mach $10700 \mathrm{~km} \mathrm{~s}^{-1}$ polytropic wind $(\gamma=4 / 3)$ with a density of $5500 \mathrm{~cm}^{-3}$.

\section{Summary of Results}

The mass accretion rate, $\dot{M}$, for a spatially uniform gas flowing into the volume of solution via a single face is larger than in earlier simulations due to different handling of the accretor boundary but agrees better with theory (Ruffert \& Melia, 1994).

We have also modeled the wind as coming from a cluster of 10 pseudorandomly placed sources. These "stars" produce expanding pockets of cool, low density gas separated by ridges of slow moving streamers of hot, dense gas that get funneled onto the accretor, increasing $\dot{M}$ and the accreted specific angular momentum, $\lambda$ (in units of $\mathrm{cR}_{s}$, where $\mathrm{R}_{s}$ is the Schwarzschild radius). Although a more compact cluster of stars produces a larger $\dot{M}$ due to more wind-wind collisions, a less compact cluster produces a somewhat larger $\lambda$ due to the more distributed nature of the wind.

For these point source models, we find an accretion rate of more than $10^{22} \mathrm{~g} \mathrm{sec}^{-1}$ (twice the uniform result) and a $\lambda$ of $40-60 \mathrm{cR}_{s}$ (5 times the uniform result), both with an RMS scatter of $\sim 15 \%$. However, the sign of the accreting angular momentum changes every $\sim 50$ years so any accretion disk, which must be less than $\sim 10^{3} \mathrm{R}_{s}$ in radius, is not likely to exist for longer than that. Finally, if Sgr A* is a single compact object powered by the Bondi-Hoyle accretion process, hydrodynamical instabilities in the flow should produce variable emission (at least $10 \%$ ) at all frequencies over a few decades; shorter timescale and larger amplitude variations are possible. Similarly, for AGN with a central engine of a given mass, the characteristics of a galaxy's nuclear activitiy (luminosity variability, the presence and type of any emission or absorption regions, the presence of an accretion disk) will be determined by the orientation and compactness of the central cluster as well as how the central cluster's winds interact with the central engine.

\section{References}

Geballe, T., Krisciunas, K., Bailey, J., and Wade, R., 1991, ApJ (Letters), 370, L73.

Ruffert, M. \& Melia, F., 1994, $A \mathcal{G} A$ (Letters), 288, L29. 